\title{
Emerging Technologies in Scar Management: Laser-Assisted Delivery of Therapeutic Agents
}

Juhee Lee and Jihee Kim

\section{Contents}

50.1 Background - 444

50.2 Laser Systems Used for Laser-Assisted Delivery - 444

50.3 Carbon Dioxide (CO2) Laser and Erbium:Yttrim-AluminumGarnet (Er:YAG) Laser - 445

50.4 Mechanism of Ablative Fractional Laser-Assisted Drug Delivery - 445

50.5 Technique and Parameters - 446

50.5.1 Main Parameters: MTZ Density and Depth - 446

50.5.2 Clinical Application in Scar Treatment: Drugs and Bioactive Molecules - 446

50.5.3 Other Modalities to Enhance the Effect of Laser-Assisted Delivery - 448

50.6 Conclusion -448

References - 449 


\subsection{Background}

Scars can cause significant impact on patients' quality life. Let alone cosmetic aspect, scars can result in functional and structural comorbidity. However, the treatment of scars is often challenging due to heterogeneity of etiologies and clinical presentations. Traditionally, surgical revision had been a mainstay for scars requiring structural remodeling to release the contracture of underlying tissue and relieve restricted range of motion. Nowadays, varieties of therapeutic options have been successfully introduced including physical therapy, silicon sheet, cryotherapy, corticosteroids, and laser modalities. Corticosteroids are often referred to as first-line treatment but its topical application is often ineffective. The major rate-limiting step for drug absorption is passage through the stratum corneum, which serves as the physiological barrier. Considering the pathogenic foci in scars are usually deep seated in the dermis, intralesional injection is considered as the best treatment option to bypass the thick stratum corneum barrier and deliver higher concentration of corticosteroids at the site. Yet the procedure inevitably accompanies excruciating pain. Corticosteroid is often associated with localized adverse events on repeated sessions causing skin atrophy, hyper or hypopigmentation, and telangiectasia. Treatment of keloid is even intriguing with varying success rates, and recurrence rates are high with conventional measures.

Target site for topically applied drug is the viable epidermis or dermis and the clinical response to a formulation is directly proportional to the concentration of the drug achieved at the target site. Transcutaneous absorption is limited by inherent skin barrier properties, minimizing the absorption to only $1-5 \%$ [1]. The innate barrier function of the skin, in particular the stratum corneum, provides the rate-limiting step in percutaneous penetration of drugs and other agents. Many different strategies have been developed to increase the permeability of the skin transiently for drug delivery. In skin with intact stratum corneum, only small $(<500 \mathrm{Da}$ in molecular weight) and lipophilic drugs succeed in partitioning into and diffusing through this dense lipidrich layer. Transdermal patches have been used since 1970s but it is limited to drugs with low molecular mass. Accordingly, there is considerable interest in developing novel drug delivery methods. To enhance topical delivery of drug, currently available physical techniques include stripping, microneedling or sonophoresis, or iontophoresis to overcome the skin barrier.

The advent of lasers, particularly fractional laser systems, significantly advanced scar treatments in past decades [2]. Laser-assisted drug delivery was first described in 1987 and initially practiced with fully ablative lasers [3]. Laser-assisted delivery has been reported and evaluated in animal models for variety of skin diseases including premalignant lesions to inflammatory conditions. Ablative lasers can be effectively used to induce absorption of medications beyond the epidermal barrier. In precancerous skin lesions such as actinic keratosis, ablative fractional laser (AFL) is used to induce penetration of photosensitizer prior to photodynamic therapy. Chemotherapeutic agents and immunomodulators like 5-fluorouracil (5-FU) or imiquimod have been successfully used. Laser-assisted drug delivery has also shown to enhance absorption of topical anesthetics, nonsteroidal anti-inflammatory drugs, and corticosteroids [4].

\subsection{Laser Systems Used for Laser-Assisted Delivery}

Ablative lasers system has been employed to improve skin penetration of active molecules. Laser devices have traditionally been used in continuous mode, in which the entirety of the water-containing epidermis is completely ablated. Elimination of the physical barrier of stratum corneum allows transcutaneous delivery of large molecules, although it requires significant recovery time after removing large areas of stratum corneum. To overcome significant drawbacks from ablative fractional resurfacing, the concept of fractional photothermolysis (FP) was proposed in 2004 [5]. Fractional photothermolysis (FP) is a technique whereby an ablative laser is administered in a fractionated pattern instead of full ablation. Fractionated systems create discreet columns of ablated tissue, known as microthermal zones (MTZs), sparing intact portion of skin. The untreated skin surrounding MTZs serves as a reservoir of growth factors and stem cells promoting tissue regeneration and wound-healing response. Accordingly, ablative fractional lasers (AFLs) have provided an emerging option with a lower side-effect profile. AFL emerged as a promising tool in the treatment of scars with minimal pain and rapid wound healing in few days [2]. Moreover, fractional laser-assisted drug delivery is employed with high precision by controlling the area and degree of ablation through laser settings. MTZs facilitate penetration of topical molecules from the surface to the layer of interest (- Fig. 50.1). Due to its predictable tissue response, it can be an effective alternative to injection or topical formulations of drugs targeting cutaneous diseases. 


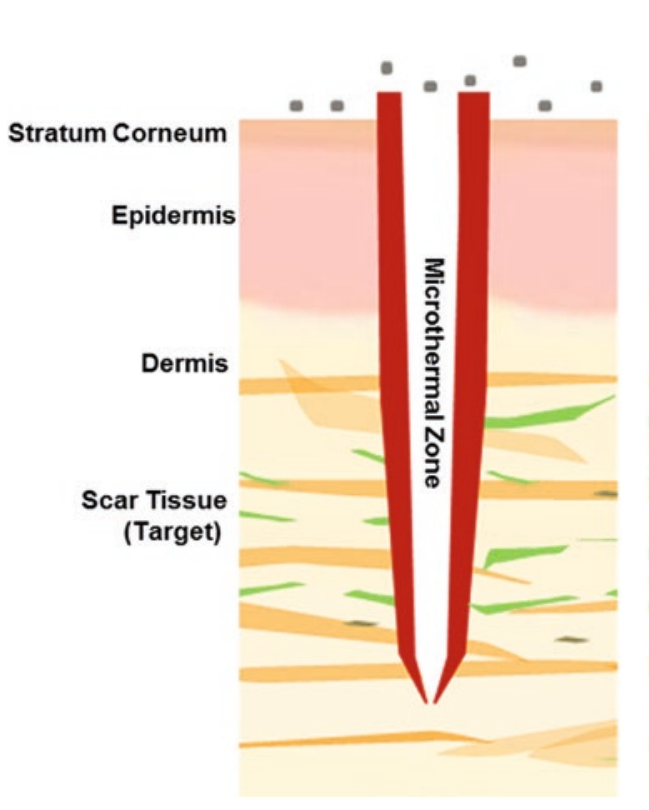

- Fig. 50.1 Schematic diagram of ablative fractional laser (AFL)assisted drug delivery. AFL irradiation creates microchannels extending from the skin surface to the dermis. Microthermal zones (MTZs) vaporize accumulated scar tissue (orange) and influence

\subsection{Carbon Dioxide $\left(\mathrm{CO}_{2}\right)$ Laser and Erbium:Yttrim-Aluminum-Garnet (Er:YAG) Laser}

Carbon dioxide $\left(\mathrm{CO}_{2}\right)$ laser and erbium:yttrim-aluminum-garnet (Er:YAG) laser are the two types of laser devices most commonly studied in regard to laserassisted drug delivery. $\mathrm{CO}_{2}$ laser $(10,600 \mathrm{~nm})$ heats cells instantly, resulting in vaporization and coagulation of irradiated cells. Subsequently, denaturation of extracellular proteins and heat-induced shrinkage of collagen is noted in a subjacent residual layer. Histologic studies of scar tissue after ablative fractional $\mathrm{CO}_{2}$ laser treatment demonstrated complete re-epithelization within 48 hours. Er:YAG laser (2940 nm) system delivers energy more precisely without extensive thermal damage to the surrounding tissue, permitting quicker wound healing and recovery of dermis. $\mathrm{CO}_{2}$ devices generate larger degrees of heat diffusion to the surrounding tissue while Er:YAG system exerts less hemostatic effect. Differences between fractional $\mathrm{CO}_{2}$ laser and fractional Er:YAG laser are similar to their full-field counterparts in that the $\mathrm{CO}_{2}$ systems cause more residual thermal damage. Studies have reported that fractional Er:YAG laser causes significantly less residual thermal dermal damage, with a faster healing time and less post-pro-

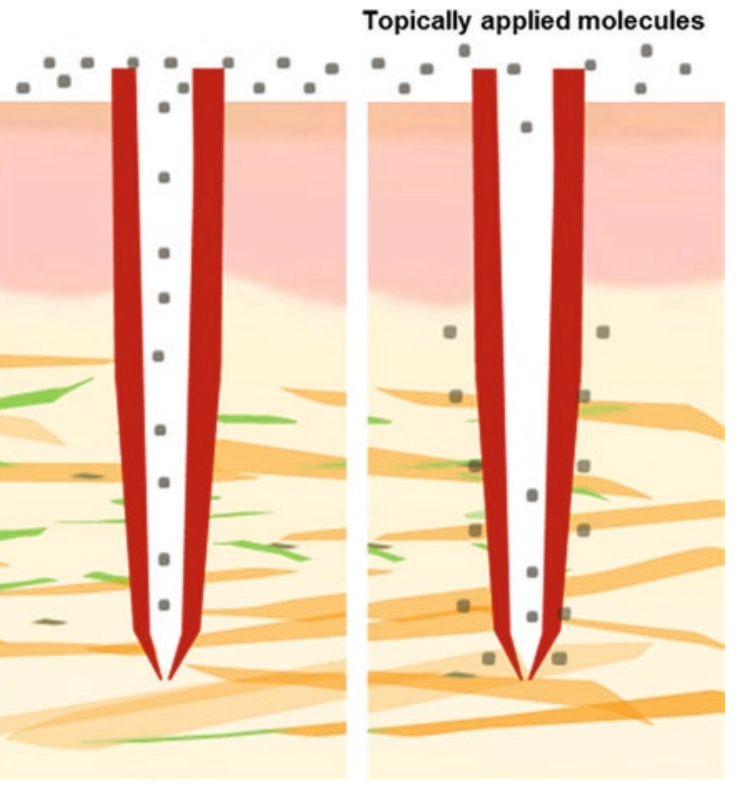

collagen remodeling (green). Microchannels transverse stratum corneum and topically applied molecules can reach target tissue in the dermis and permeate into the skin

cedure erythema compared to fractional $\mathrm{CO}_{2}$ laser [4]. When applicable, the combined-mode Er:YAG/ $\mathrm{CO}_{2}$ laser system can offer synergistic benefit of ablative and coagulation effect. Both lasers have been successfully applied for scar treatment with favorable clinical outcomes. Fractional $\mathrm{CO}_{2}$ and Er:YAG provided comparable outcome, yet fractional $\mathrm{CO}_{2}$ system was associated with a higher degree of procedural pain in few studies [6]. However, it is difficult to determine superiority between the two modalities due to clinical heterogeneity and lack of comparative study design.

\subsection{Mechanism of Ablative Fractional Laser-Assisted Drug Delivery}

Ablative fractional lasers irradiate high-fluence laser beam forming multiple, discrete columns of thermal damage. Ablative fractional photothermolysis (AFP) systems generate MTZs by vaporizing full thickness zones of scar tissue that may extend to deep dermis. Surrounding the denatured columns created by MTZs is a collateral area of thermal denaturation that is sufficient to coagulate collagen. Moreover, thermal irradiation stimulates neocollagenesis for collagen remodeling. Studies have demonstrated that the increased expres- 
sion of heat shock proteins (HSPs) induces antiinflammatory effect after ablative laser treatment for scars. Additionally, the altered expressions of matrix metalloproteinases (MMPs) and tissue inhibitors of metalloproteinases (TIMPs) are noted along with increased expression of growth factors. Collectively, the differential expression of anti-inflammatory mediators and cytokines attribute to improvement in clinical appearance of scars.

MTZs facilitate penetration of topically applied drugs and other bioactive agents by acting as an alternative pathway for cutaneous drug delivery. Increased penetration of molecule can be explained by Fick's first law of diffusion. The diffusive flux of molecule goes from regions of high concentration to lower concentration, with a magnitude that is proportional to the concentration gradient. The degree of flux $(J)$ is described as a product of partition coefficient $(K)$, diffusion coefficient $(D)$, and concentration difference across the barrier $(\Delta C)$ divided by the diffusion distance $(L)$. Partition coefficient $(K)$ indicates the equilibrium solubility of the drug molecules available for diffusion across a membrane.

$$
J=\frac{K \times D \times \Delta C}{L}
$$

The diffusion coefficient reflects the measure of inherent diffusivity of a molecule and determines the diffusion rate once the molecule permeates the membrane. The diffusion distance represents the length of a path for the diffusion. MTZs generated by AFL impact flux in various aspects of Fick's first-law variables. Firstly, increased permeability via MTZs enables increase in $K$ compared to intact skin. Secondly, larger molecules with higher diffusivity can permeate directly into epidermis. Lastly, the distance of the diffusion is decreased from the skin surface and enables delivery to deeper layer. As a result, pretreatment with AFL facilitates traditionally challenging uptake of large hydrophilic molecules. The use of AFL increases the transdermal drug delivery of large molecules by 8 - to 15 -fold [4].

\subsection{Technique and Parameters}

\subsubsection{Main Parameters: MTZ Density and Depth}

AFL system irradiates target scar tissue with high precision by controlling the area and degree of ablation through laser settings such as power, pulse duration, percentage of skin coverage, and ablation pattern. For AFL-assisted drug delivery, the density and depth of microablative columns should be determined by operator for the given clinical characteristics of the scars. MTZs formed by AFP system can be adjusted to tar- get specific depth and diameter by increasing the fluence and induce regulated disruption of epidermal barrier. In general, relatively modest energy fluence and density are considered applicable for laser-assisted delivery. Low energy levels are classically all that are needed to impair the physical barrier of stratum corneum. By calibrating laser settings, it is possible to influence the delivery rate and drug biodistribution, which may lead to improved clinical efficacy with shortened incubation time. Studies of the effect of laser parameters on the transdermally absorbed molecules are limited.

\subsubsection{MTZ Density and Coverage}

The MTZs consist of sharply confined tissue denaturation with a diameter of about $100 \mu \mathrm{m}$ at intervals of about $200 \mu \mathrm{m}$. In animal models, increased density of MTZs achieved overall drug delivery, yet there was threshold density to achieve maximum diffusion. Fluorescent labeling demonstrated that the absorption of topically applied photosensitizers, immunomodulators, and lidocaine reached maximum biodistributions of $5 \%, 10 \%$, and up to $20 \%$, respectively, while further increase only resulted in deposition to stratum corneum [3]. More importantly, complications, such as scarring and hypopigmentation, have been observed at coverages in excess of $45 \%$.

\subsubsection{MTZ Depth and Energy}

The depth of ablation represents how deep the MTZs extend into the skin and for a given laser beam diameter is mainly controlled by laser pulse energy. Microbeams of AFL irradiation typically induce MTZs ranging from 100 to $300 \mu \mathrm{m}$ in diameter and it can extend down to the deep reticular dermis. The optimal depth threshold is yet undetermined as studies report drug deposition as both dependent on and independent of laser channel depth. It is expected that increase in the laser fluence and irradiation time increases the cellular uptake of large molecules across the skin in a dose-dependent manner. The usual threshold in the literature is approximately 200-250 $\mu \mathrm{m}$ in diameter. Hydrophilic or semi-lipophilic molecules (methotrexate, prednisone, and diclofenac) are generally dependent on MTZ depth while lipophilic molecules (lidocaine, ingenol mebutate, and imiquimod) do not show obvious depth-dependent uptake [6]. The variation between optimal depths for penetration can be related to the location of vascular plexus in different types of skin used for evaluation.

\subsubsection{Clinical Application in Scar Treatment: Drugs and Bioactive Molecules}

Delivery of every molecule will vary based on its inherent properties such as size, diffusion coefficient, and individuals' predisposed skin barrier conditions. In 
stratum corneum, corneocytes are embedded in a lipid matrix and bioavailability of hydrophilic drugs is low. While lipophilic or semi-lipophilic molecules can penetrate lipid barrier of the outmost layer of the epidermis, large or hydrophilic molecules are able to transverse the stratum corneum after AFL pretreatment.

\subsubsection{Corticosteroid}

Corticosteroid has been a mainstay in the scar treatment. It is mostly known to inhibit inflammatory mediators and promotes remodeling of extracellular matrix. To exert its clinical effect, corticosteroid should penetrate cell membrane to bind the nuclear receptor. Sustained release of corticosteroid can be beneficial because wound regeneration of the inflammatory stages is known to persist for several days after laser irradiation. Triamcinolone acetonide is the most widely used and available as micronized suspensions of corticosteroid crystals. Currently, intralesional injection of corticosteroid has been well advocated in the treatment of both hypertrophic scars and keloids. After its administration, micronized crystals of corticosteroids persist in skin and are released over a period of weeks. However, local injection of triamcinolone actinide is often painful and consistent dosing is difficult to achieve throughout the scar.

Combination treatment of AFL and topically applied corticosteroids demonstrate encouraging results. Application of triamcinolone immediately after AFL can induce synergistic effect of deep penetration into the scar tissue to induce collagen remodeling. Use of relatively low-dose triamcinolone $(10 \mathrm{mg} / \mathrm{mL})$ is sufficient to induce clinical improvement in various types of scars including burn, trauma, or surgical scars. AFL-assisted delivery of topical triamcinolone potentially offers an efficient, safe, and effective adjunctive treatment. Corticosteroids available as topically applied formulations demonstrated mediocre effect in scar treatment. There are only few reports used to alleviate symptoms or appearance of hypertrophic scars immediately after surgical procedure [7]. Nevertheless, the combination of AFL and topical corticosteroid application yields successful result for the treatment of keloid without any adverse event [8]. AFL-assisted corticosteroid application is a promising tool with minimal pain and rapid wound healing in few days. Beyond the specific action of the lasers on scar remodeling, the channels that they create can be used to deliver active molecules to potentiate its efficacy (- Fig. 50.2). On the other hand, the presence of MTZ-induced channels is transient mostly at presence for 3 to 7 days. Accordingly, disrupted physical barrier of stratum corneum is restored within a week after AFL. Thus, further study is required to determine the optimal dosage of triamcinolone suspension and potency of topically applied formulations.

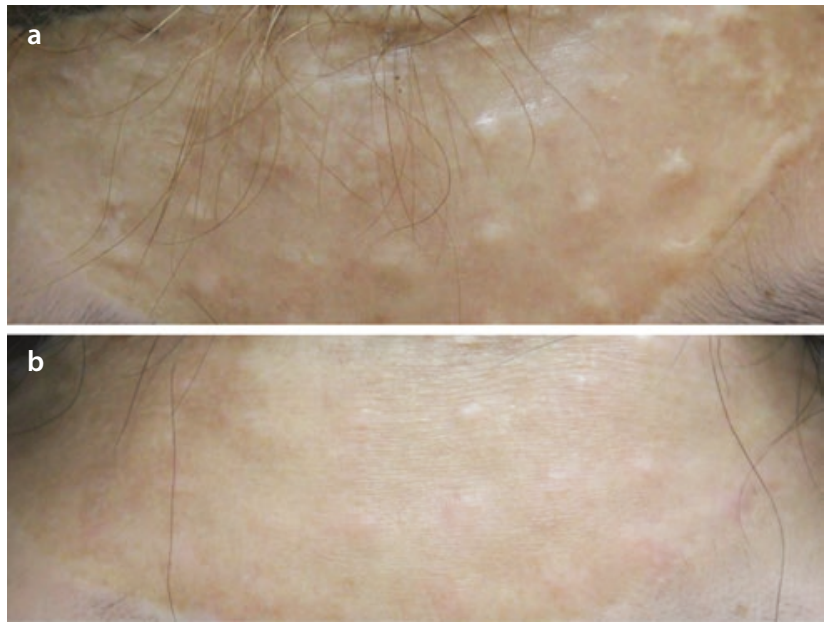

- Fig. 50.2 a A 16-year-old woman with Fitzpatrick skin type IV with hypertrophic scars after facial reconstruction surgery. b Patient underwent five sessions of fractional $\mathrm{CO}_{2}$ laser $\left(\mathrm{eCO}_{2}\right.$ Lutronic, Goyang, Korea. $60 \mathrm{~mJ}, 10 \%$ density) combined with topical application of triamcinolone acetonide suspension of $10 \mathrm{mg} / \mathrm{mL}$

\subsubsection{Other Agents for Scar Treatment}

When routinely applied, topical corticosteroids do have risk to cause localized adverse effects such as skin atrophy, pigmentary changes, or telangiectasia. To spare such undesired skin reaction, drugs with immunomodulatory function has been applied in various skin conditions.

5-Fluorouracil (5-FU) is a pyrimidine analog antimetabolite that results in an irreversible inhibition of thymidylate synthase, commonly used for premalignant skin lesions. Laser-assisted delivery of 5-FU has been tested in animal models using various types of laser systems. Pretreatment with AFL resulted in more than 50-fold and 30-fold increase in penetration using Er:YAG and $\mathrm{CO}_{2}$ system, respectively. In a study comparing the effect of 5-FU and triamcinolone to treat hypertrophic scars, lesions subjected to 5-FU demonstrated comparable results in terms of scar texture and height [9]. Appropriate dosing of 5-FU should be evaluated due to concern for increased systemic absorption with laser-assisted drug delivery, which can expand the adverse effect profile unlike bland topical application.

Atrophic scars result from loss of dermal or subcutaneous tissue during the wound-healing process. Fillers are high-molecular-weight biopolymer developed to provide structural support for volume restoration of skin. On this account, it is unable to permeate through intact epidermis and employed by injection. Laser systems, AFL in particular, can improve atrophic scars by promoting new collagen synthesis after irradiation. Concomitant use of AFL and topically applied polyL-lactic acid (PLLA) fillers proved to be successful in treating patients with atrophic scars from various eti- 
ologies including acne and trauma. Histologic evaluation on cadaveric skin demonstrated the penetration of PLLA to the dermis, albeit large molecular size [10]. Additionally, sustained release by prednisolone encapsulated in PLLA microsphere provided continuous delivery enough to suppress prolonged inflammatory response [6]. Likewise, several bioactive molecules and peptides such as polydeoxyribonucleotide (PDRN) are evaluated for potential application for scar treatment. Recently, enhanced percutaneous delivery of adipocyte and hematopoietic stem cells by AFL systems has been evaluated in scar management.

\subsubsection{Other Modalities to Enhance the Effect of Laser-Assisted Delivery}

\subsubsection{Emerging Devices}

AFL-assisted drug delivery of therapeutic agents emerging devices is one of the latest strategies to enhance cutaneous bioavailability of topically applied molecules. Notwithstanding that MTZs can empower to bypass the skin barrier, passive uptake through MTZs can be inadequate as the channels are rapidly restored within few days. Furthermore, within few hours after ablation, the depth of columns is gradually shortened by accumulated fibrin and interstitial granulation tissue. Therefore, several physical maneuvers have been suggested to enhance the delivery of active molecules. Ultrasound may temporarily reduce the intact biding of stratum corneum. Acoustic waves by ultrasound may induce cavitation, which increases the penetration of molecules. In animal model, active filling of MTZs was observed by altering the pressure during the topical application of test molecule [6]. Similarly, combination of sonophoresis and iontophoresis with AFLassisted drug delivery can induce favorable results.

Radiofrequency (RF) devices locally generate heat depending on the local electrical resistance and current density. RF devices deliver thermal energy to the dermis and subdermal tissue, which induces collagen contraction and remodeling by disrupting its structural bond, thereby most commonly applied to the treatment of facial rhytides and skin tightening. Advances in laser and energybased devices expanded clinical indications of RF devices. Fractionated RF systems are used to improve scar texture, and bipolar fractional microneedle RF systems have shown the resolution of atrophic acne scars. Furthermore, ablative fractional RF associated with acoustic pressure generated by ultrasound was used to increase the delivery of triamcinolone into hypertrophic scars [8].

The use of non-ablative fractional lasers (NAFL) needs to be examined as a less invasive method of laserassisted drug delivery. NAFL systems create a similar array of microablative columns as does AFLs; the MTZs consist of columns of thermal injury with intact overlying stratum corneum. NAFL systems heat tissue without vaporization, thus being less associated with patient discomfort. Although the epidermis is not fully vaporized, barrier function of the skin in the treated area is transiently impaired allowing for enhanced drug delivery. NAFLs applied alone have confirmed its therapeutic efficacy in skin rejuvenation and various types of scars. NAFL-assisted drug delivery of minoxidil or corticosteroids can be applied to treat alopecia areata. Fractional erbium glass laser $(1550 \mathrm{~nm})$ was reported to effectively enhance percutaneous delivery of photosensitizers [4].

\subsubsection{Limitations}

Further investigations are required to establish the safety and efficacy of laser-assisted delivery of molecules as a transcutaneous drug delivery system. The majority of the existing studies have been performed on animal models and additional human studies are needed. Drug permeation through AFL-generated MTZs inevitably raises concern about systemic absorption and potential systemic toxicity. Transdermal delivery is considered relatively safe as the dosage required for the therapeutic effect of a drug is lower than the oral dose and avoids the drug metabolism in the liver. Although most applications of laser-assisted drug delivery have been intended for local effects in the skin, some reports have demonstrated that systemic absorption does occur. Along with increased transcutaneous absorption, a decreased dose should be considered to minimize side-effects that could be local or systemic. Further clinical studies are needed to fully evaluate the safety and efficacy of laser-assisted drug delivery for scar treatment.

\subsection{Conclusion}

Laser-assisted drug delivery is an evolving technology with many possible applications as a highly targeted customizable method for distribution of drugs within the skin. Current studies have demonstrated that laser pretreatment of the skin can increase the permeability and depth of penetration of topically applied drug molecules. Fractional laser systems can be successfully utilized in the treatment of various forms of scarring with a very favorable safety profile. Fractional photothermolysis, both ablative and non-ablative, can improve the texture of various scars by promoting collagen remodeling. AFL-assisted drug delivery systems offer distinct advantage by enabling the topical medication to penetrate deeper and reach target pathogenic site even for the systemic drug administration. Corroborative efforts with multidisciplinary measure and novel combinations of existing treatments can help ensure optimal clinical and cosmetic results. 


\section{Take-Home Message}

- Lasers are safe and effective means of enhancing the delivery of topically applied agents through the skin.

- Ablative fractional laser-assisted drug delivery is increasingly used to enhance percutaneous uptake.

- Microthermal zones generated by fractional laser create precise, uniform columns of tissue vaporization, which facilitates delivery of various drugs.

- Ablative fractional laser-assisted corticosteroid delivery may take advantage of the newly formed channels to penetrate uniformly and deeply into the scar tissue. Combination of valuable scar treatment options can create synergistic therapeutic response.

- Laser-assisted drug delivery provides a new opportunity for a minimally invasive modality in scar treatment.

\section{References}

1. Sandberg C, Halldin CB, Ericson MB, Larko O, Krogstad AL, Wennberg AM. Bioavailability of aminolaevulinic acid and methylaminolaevulinate in basal cell carcinomas: a perfusion study using microdialysis in vivo. Brit J Dermatol. 2008;159(5):1170-6. https://doi.org/10.1111/j.13652133.2008.08795.x.

2. Anderson RR, Donelan MB, Hivnor C, Greeson E, Ross EV, Shumaker PR, et al. Laser treatment of traumatic scars with an emphasis on ablative fractional laser resurfacing consensus report. JAMA Dermatol. 2014;150(2):187-93. https://doi. org/10.1001/jamadermatol.2013.7761.

3. Haedersdal M, Erlendsson AM, Paasch U, Anderson RR. Translational medicine in the field of ablative fractional laser (AFXL)assisted drug delivery: a critical review from basics to current clinical status. J Am Acad Dermatol. 2016;74(5):981-1004. https://doi.org/10.1016/j.jaad.2015.12.008.

4. Sklar LR, Burnett CT, Waibel JS, Moy RL, Ozog DM. Laser assisted drug delivery: a review of an evolving technology. Laser Surg Med. 2014;46(4):249-62. https://doi.org/10.1002/ 1sm.22227.

5. Manstein D, Herron GS, Sink RK, Tanner H, Anderson RR. Fractional photothermolysis: a new concept for cutaneous remodeling using microscopic patterns of thermal injury. Laser Surg Med. 2004;34(5):426-38. https://doi.org/10.1002/ $1 \mathrm{sm} .20048$.

6. Ali FR, Al-Niaimi F. Laser-assisted drug delivery in dermatology: from animal models to clinical practice. Laser Med Sci. 2016;31(2):373-81. https://doi.org/10.1007/s10103-015-1853-z.

7. Park JH, Chun JY, Lee JH. Laser-assisted topical corticosteroid delivery for the treatment of keloids. Laser Med Sci. 2017;32(3):601-8. https://doi.org/10.1007/s10103-017-2154-5.

8. Cavalie M, Sillard L, Montaudie H, Bahadoran P, Lacour JP, Passeron T. Treatment of keloids with laser-assisted topical steroid delivery: a retrospective study of 23 cases. Dermatol Ther. 2015;28(2):74-8. https://doi.org/10.1111/dth.12187.

9. Waibel J, Wulkan A. Treatment of hypertrophic scars using laser assisted corticosteroids vs laser assisted 5-fluoruracil delivery. Laser Surg Med. 2013;45:14.

10. Rkein A, Ozog D, Waibel JS. Treatment of atrophic scars with fractionated $\mathrm{CO} 2$ laser facilitating delivery of topically applied poly-L-lactic acid. Dermatol Surg. 2014;40(6):624-31. https:// doi.org/10.1111/dsu.0000000000000010.

Open Access This chapter is licensed under the terms of the Creative Commons Attribution 4.0 International License (http://creativecommons. org/licenses/by/4.0/), which permits use, sharing, adaptation, distribution and reproduction in any medium or format, as long as you give appropriate credit to the original author(s) and the source, provide a link to the Creative Commons license and indicate if changes were made.

The images or other third party material in this chapter are included in the chapter's Creative Commons license, unless indicated otherwise in a credit line to the material. If material is not included in the chapter's Creative Commons license and your intended use is not permitted by statutory regulation or exceeds the permitted use, you will need to obtain permission directly from the copyright holder.

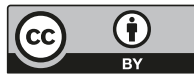

